UVX 2010 (2011) 187-191

DOI: $10.1051 /$ uvx/2011026

(C) Owned by the authors, published by EDP Sciences, 2011

\title{
Controverse sur la technique de génération d'harmoniques dite XPA
}

\author{
S. Daboussi ${ }^{1,2}$, S. Kazamias ${ }^{1,2}$, O. Guilbaud ${ }^{1,2}$, K. Cassou $^{1,2}$, C. Montet ${ }^{1}$, \\ O. Neveu ${ }^{1,2}$, B. Cros $^{1,2}$, D. Ros ${ }^{1,2}$ et G. Maynard ${ }^{1}$ \\ ${ }^{1}$ LPGP, Univ. Paris-Sud, UMR 8578, CNRS, Bât. 210, Campus d'Orsay, 91405 Orsay \\ Cedex, France \\ ${ }^{2}$ LASERIX, CLUPS, Univ. Paris-Sud, CNRS, Campus d'Orsay, 91405 Orsay Cedex, France
}

\begin{abstract}
Résumé. Un article est récemment paru dans le journal "Nature Physics" sous le nom "Laser-driven amplification of soft X-rays by parametric stimulated emission in neutral gases" [1]. Suite à l'observation expérimentale de la croissance exponentielle du signal harmonique en fonction de la pression de gaz, les auteurs annoncent que seule une nouvelle théorie qu'ils appellent "XPA" pour (X-ray parametric amplification) permet d'expliquer le résultat. Or dans ce qui suit nous montrons qu'une étude détaillée de l'accord de phase dans le cadre de la théorie classique de la génération d'harmoniques d'ordres élevés permet de reproduire parfaitement et d'expliquer la croissance exponentielle du signal harmonique en fonction de la pression par l'augmentation de la longueur de cohérence due à la dispersion atomique.
\end{abstract}

\section{INTRODUCTION}

La génération d'harmoniques d'ordres élevés (HHG en abréviation anglaise) est un processus hautement non-linéaire induit dans les gaz rares par un champ laser très intense dans le régime femtoseconde. L'efficacité de ce processus résulte de la combinaison de deux aspects:

1) L'aspect microscopique et la réponse de l'atome unique:

L'émission harmonique est due à une réponse fortement non-linéaire des atomes d'un gaz soumis à un champ laser intense. Ce phénomène peut être décrit par un modèle dit à trois étapes qui a été analysé pour la première fois en 1992 en utilisant une approche semi-classique [2,3]. En 1994 une étude quantique complète a été effectuée par Lewenstein dans le cadre de l'approximation du champ fort (SFA) [4]. Ce modèle permet de calculer la phase et l'amplitude du dipôle harmonique en fonction de l'intensité laser et des autres paramètres de génération. Les trois étapes sont les suivantes: Ionisation par effet tunnel induite par le laser, accélération de l'électron quasi libre dans le champ laser et finalement recombinaison atome-électron avec émission d'un photon harmonique.

2) L'aspect macroscopique et le problème de l'accord de phase:

Le signal harmonique total est la superposition cohérente des champs émis par les atomes du gaz le long de la propagation dans la cellule de génération. Le signal en sortie de cellule est maximal quand l'accord de phase est obtenu, c'est à dire quand les dipôles harmoniques de la cellule sont en phase. Pour cela, il faut que les vitesses de propagation du laser infrarouge et des harmoniques soient égales. Dans le cas contraire on appelle longueur de cohérence la distance dans la cellule qui sépare deux dipôles harmoniques avec une différence de phase de $\pi$, qui vont donc interférer destructivement.

This is an Open Access article distributed under the terms of the Creative Commons Attribution-Noncommercial License 3.0, which permits unrestricted use, distribution, and reproduction in any noncommercial medium, provided the original work is properly cited. 


\section{MODÉLISATION 1D DE LA GÉNÉRATION D'HARMONIQUES D’ORDRES ÉLEVÉS}

La signal harmonique instantané $S_{H H G}(t)$ à la sortie du milieu dépend de la longueur du milieu $l_{\text {med }}$, de la longueur d'absorption $l_{a b s}$ et de la longueur de cohérence $l_{c o h}$ selon la loi [5]:

$$
S_{H H G}(t) \propto P^{2} A_{q}^{2} \frac{4 l_{a b s}^{2}}{1+4 \pi^{2}\left(l_{a b s}^{2} / l_{c o h}^{2}\right)} \times\left[1+e^{-l_{\text {med }} / l_{a b s}}-2 \cos \left(\pi l_{\text {med }} / l_{\text {coh }}\right) e^{-l_{\text {med }} / 2 l_{a b s}}\right]
$$

avec P la pression et $A_{q}$ l'amplitude du dipôle harmonique. La longueur de cohérence dépend fortement de l'ionisation du milieu qui fixe les dispersions atomique et électronique. La longueur de cohérence est donnée par $l_{c o h}=\frac{\pi}{|\delta k|}$ avec

$$
\delta k=(q \omega / c)\left(\delta n_{a t}+\delta n_{\text {elec }}\right)+q \delta k_{\text {Gouy }}+\delta k_{\phi_{a t}}
$$

q est l'ordre harmonique, $\omega$ est la pulsation laser, c la célérité de la lumière, $\delta k_{\text {Gouy }}$ la dispersion de Gouy qui dépend des conditions de focalisation du laser, $\delta k_{\phi_{a t}}$ est le gradient de la phase atomique qui provient de la réponse de l'atome unique.

Nous avons développé un code unidimensionnel permettant d'étudier la dépendance en fonction du temps des paramètres harmoniques [6]. Nous avons calculé le signal des harmoniques H31 et H55 pour l'argon en utilisant comme paramètres d'entrée l'intensité laser (de 1 à $\left.3 \times 10^{14} \mathrm{~W} / \mathrm{cm}^{2}\right)$, la durée d'impulsion ( $350 \mathrm{fs})$, la longueur d'onde du laser $\left(\lambda_{\text {laser }}=1054 \mathrm{~nm}\right)$, la longueur de Rayleigh $(5 \mathrm{~mm})$ et la longueur du milieu $(2 \mathrm{~mm})$. Les longueurs d'absorption qui varient comme l'inverse de la pression sont récapitulées dans ce tableau pour une pression 100 mbar d'argon [7].

\begin{tabular}{|l|l|l|l|l|l|l|l|l|l|l|l|l|l|}
\hline HHG & 31 & 33 & 35 & 37 & 39 & 41 & 43 & 45 & 47 & 49 & 51 & 53 & 55 \\
\hline$l_{\text {abs }(\mathrm{mm})}$ & 0.23 & 0.60 & 1.34 & 2.72 & 4.03 & 4.45 & 4.65 & 4.49 & 4.25 & 3.98 & 3.68 & 3.40 & 3.18 \\
\hline
\end{tabular}

Les dispersions atomique et électronique dans l'Argon pour $\lambda_{\text {laser }}=1054 \mathrm{~nm}$ sont données par

$$
\delta k_{\text {atomic }}\left[\mathrm{mm}^{-1}\right]=-q \cdot 1.66 \times 10^{-3} \cdot P[\mathrm{mbar}] \cdot(1-\tau)
$$

et

$$
\delta k_{\text {electronic }}\left[\mathrm{mm}^{-1}\right]=q(0.072 \cdot \tau \cdot P[\mathrm{mbar}])
$$

avec $\tau$ le degré d'ionisation de l'Argon calculé en utilisant les taux ADK [8].

Le gradient de phase atomique qui joue un rôle important dans l'accord de phase est exprimé pour les deux chemins quantiques par

$$
\delta k_{\phi_{a t}}\left[\mathrm{~mm}^{-1}\right]=\alpha \nabla I
$$

avec $\alpha=2 \times 10^{-14} \mathrm{~cm}^{2} / \mathrm{W}$ pour le premier chemin quantique et $\alpha=22 \times 10^{-14} \mathrm{~cm}^{2} / \mathrm{W}$ pour le second chemin quantique [9].

Le calcul de ces paramètres en fonction du temps permet de déterminer la valeur de $l_{c o h}(t)$ et du signal harmonique. Le signal est ensuite intégré sur toute la durée d'impulsion séparément pour les chemins 1 et 2. $S_{H H G}(t)$ total est la somme des deux contributions.

\section{RÉSULTATS ET DISCUSSIONS}

\subsection{Influence de la longueur de cohérence sur le signal harmonique}

La figure 1 présente la variation du signal total des harmoniques $\mathrm{H} 31$ et $\mathrm{H} 55$ en fonction de la pression pour trois valeurs d'intensités laser : $1,1.5$ et $2 \times 10^{14} \mathrm{~W} / \mathrm{cm}^{2}, z_{0}=5 \mathrm{~mm}$ et $z_{\text {cell }}=2 \mathrm{~mm}$. Cette figure montre que nos simulations sont en bon accord avec les résultats expérimentaux publiés dans [1], le signal harmonique croît bien exponentiellement avec la pression pour une large gamme d'intensités 
sur plusieurs ordres de grandeur jusqu'à une valeur d'environ 100 mbar. On reproduit également le fait qu'une saturation du signal se produit à haute pression et haute intensité alors que le signal chute à haute pression basse intensité. Notre modèle ne permet pas la défocalisation du laser qui pourrait intervenir à haute pression et haute intensité.
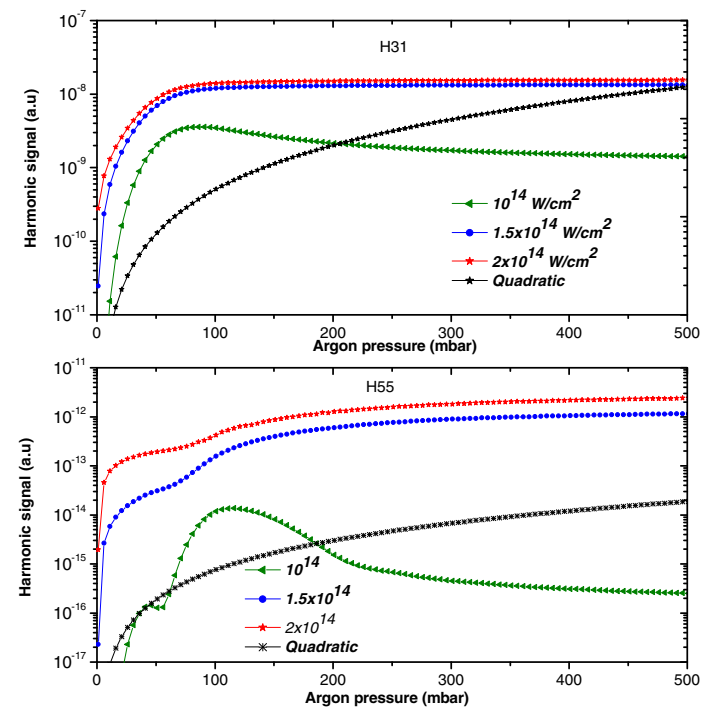

Figure 1. Signal des harmoniques $\mathrm{H} 31$ et $\mathrm{H} 55$ en fonction de la pression pour trois valeurs d'intensité laser : respectivement $1(\triangleleft), 1.5(\bullet)$ and $2(\star) 10^{14} \mathrm{~W} / \mathrm{cm}^{2}$.

Pour expliquer l'origine de ces comportements nous avons étudié l'évolution de $l_{c o h}$ et du signal harmonique en fonction de la pression pour une basse intensité laser (ionisation faible dans le milieu $(\tau \sim 0)$ ) ; dans ces conditions la longueur de cohérence s'exprime comme :

$$
\begin{aligned}
& l_{\text {coh }} \simeq \frac{\pi}{\left|\delta k_{\text {Gouy }}+\delta k_{\text {atomic }}\right|} \\
& \simeq \frac{\pi / q}{\left|\frac{1}{z_{0}+\frac{z_{\text {cell }}^{2}}{z_{0}}}-P \cdot 1.66 \cdot 10^{-3}\right|}
\end{aligned}
$$

La figure 2 présente la variation analytique de $l_{c o h}(\tau \sim 0)$ pour une basse intensité laser $\left(10^{14} \mathrm{~W} / \mathrm{cm}^{2}\right)$ (équation (7)) et de $S_{H H G}(\tau \sim 0)$ (équation (1)) en fonction de la pression pour H31, $z_{0}=5 \mathrm{~mm}$ et $z_{\text {cell }}=2 \mathrm{~mm}$. Une augmentation importante de $l_{\text {coh }}$ est observée jusqu'à $\mathrm{P}=100 \mathrm{mbar}$ suivie d'une forte décroissance. Ce comportement dû à la dispersion atomique explique parfaitement les résultats obtenus.

Le signal harmonique croît avec la pression et atteint sa valeur maximale pour une pression égale à 100 mbar pour laquelle la dispersion atomique compense la phase de Gouy, d'où le meilleur accord de phase. Pour des valeurs de pression plus élevées, le signal décroît suite à la décroissance de $l_{c o h}$.

\subsection{Interférences destructives entre les dipôles harmoniques}

Nous avons aussi montré que notre modèle permet de reproduire les inflexions observées dans le signal harmonique à basse intensité basse pression (comme le montre la figure 1 pour H55 et pour une ionisation dans le milieu $\tau \sim 0$ ). Au cours de son augmentation avec la pression, la longueur de 


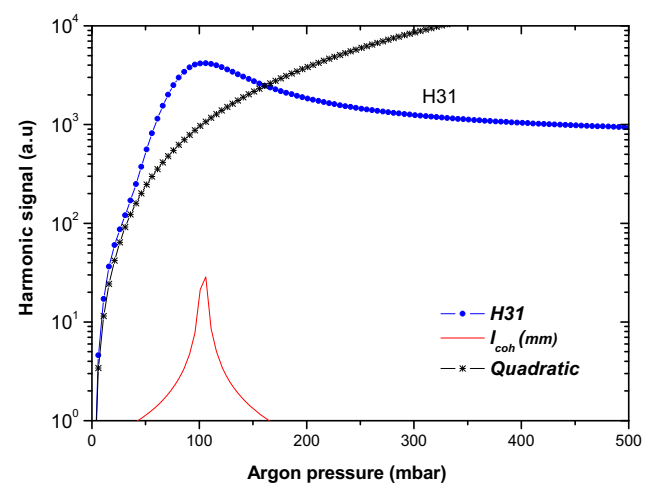

Figure 2. Signal de l'harmonique H31 en fonction de la pression (•), courbe de croissance quadratique (*), longueur de cohérence en mm.

cohérence passe par des valeurs pour lesquelles elle devient un sous multiple pair ou impair de la longueur du milieu. Or en regardant le terme $\cos \left(\pi l_{\text {med }} / l_{c o h}\right)$ de l'équation (1) on remarque que le flux harmonique se construit pour les longueurs de cohérence qui sont des sous multiples impairs de $l_{\text {med }}$ et se détruit pour les sous multiples pairs [11]. L'étude de l'évolution analytique de la longueur de cohérence montre que pour une certaine valeur de pression, $l_{c o h}$ passe par la valeur $1 \mathrm{~mm}$ (la longueur du milieu est $l_{\text {med }}=2 \mathrm{~mm}$ ). Pour cette valeur de pression il se produit des interférences destructives entre les dipôles harmoniques, ce qui arrête l'augmentation du signal. Dès que la pression ré-augmente, la longueur de cohérence s'éloigne de la valeur $1 \mathrm{~mm}$ donc le signal reprend sa croissance exponentielle.

\section{CONCLUSION}

Dans ce travail nous avons montré que la théorie classique de la génération d'harmoniques d'ordres élevés permet de reproduire et d'expliquer la croissance exponentielle du signal harmonique en fonction de la pression dans les conditions expérimentales présentées dans la référence [1]. La croissance de la pression fait augmenter la dispersion atomique qui vient à son tour compenser la forte focalisation $\left(z_{0}=5 \mathrm{~mm}\right)$, d'où l'augmentation de la longueur de cohérence signe d'accord de phase dans le milieu. Ceci provoque l'augmentation du nombre de photons harmoniques. Ce travail à fait l'objet d'un commentaire publié dans le journal "Nature Physics" [10] et d'un article soumis à "New Journal of Physics" où nous avons présenté une étude plus détaillée de l'accord de phase dans la génération d'harmoniques en fonction de la pression [12].

\section{Références}

[1] J. Seres, E. Seres, D. Hochhaus, B. Ecker, D. Zimmer, V. Bagnoud, T. Kuehl et C. Spielmann, Nature Physics 6, 455 (2010)

[2] P.B. Corkum, Phys. Rev. Lett. 71, 1994 (1993)

[3] J.L. Krause, K.J. Schafer et K.C. Kulander, Phys. Rev. Lett. 683535 (1992)

[4] M. Lewenstein, Ph. Balcou, M.Yu. Ivanov, A. LHuillier, et P.B. Corkum, Phys. Rev. A 492117 (1994)

[5] E. Constant, D. Garzella, P. Breger, E. Mével, Ch. Dorrer, C. Le Blanc, F. Salin, et P. Agostini, Phys. Rev. Lett. 821668 (1999)

[6] S. Kazamias, D. Douillet, F. Weihe, C. Valentin, A. Rousse, S. Sebban, G. Grillon, F. Augé, D. Hulin, et Ph. Balcou, Phys. Rev. Lett. 90193901 (2003)

[7] CXRO website: www.cxro.lbl.gov 
[8] Delone et al, Physics Uspekhi 40469 (1998)

[9] Ph. Balcou, A.S. Dederichs, M.B. Gaarde et A. L'Huillier, J. Phys. B, 322973 (1999)

[10] S. Kazamias, S. Daboussi, O. Guilbaud, K. Cassou, C. Montet, O. Neveu, D. Ros, B. Cros et G. Maynard, Nature Physics (2010)

[11] S. Kazamias, D. Douillet, C. Valentin, F. Weihe, F. Augé, Th. Lefrou, G. Grillon, S. Sebban, et Ph. Balcou, Phys. Rev. A, 68033819 (2003)

[12] S. Kazamias, S. Daboussi, O. Guilbaud, K. Cassou, D. Ros, B. Cros et G. Maynard, à soumettre à New Journal of Physics (2010) 